\title{
Title: Cardiac Rehabilitation: A Euro Postcode Lottery?
}

\section{Authors:}

Stephen J. Leslie, Consultant Interventional Cardiologist, Raigmore Hospital, Inverness

Neil J. Angus, Senior Lecturer in Adult Nursing, University of Stirling

Kotseva and colleagues recently published details of a large cross-sectional survey focussing on the use and effects of cardiac rehabilitation programmes (CRPs) in European countries [Kotseva et al. 2012]. The findings demonstrated considerable clinical variation in the provision and uptake of CRPs and concluded that programmes in Europe are underused, have poor referral and low participation rates with wide international variation. Despite these apparent deficiencies the study group noted improved outcomes in groups attending CR specifically in relation to smoking behaviour, cholesterol control and the use of cardioprotective medication.

It is abundantly clear from this study that CR is not currently delivered in a consistent, comprehensive or equitable manner. This no doubt reflects the considerable differences that exist between countries in the organisation and financing of health care. These differences are likely to persist and the European financial backdrop is unlikely to improve any time soon.

While CR has been reported to be a relatively inexpensive and cost effective intervention a widespread failure to fully integrate $C R$ in clinical services is still apparent - why is this, and why has provision of CR not been addressed in the same way as angioplasty services, which are arguably much more expensive and challenging to implement? Workers in the CR field and enthusiastic supporters of CR continue to make much of the evidence in support of CR and it is stressed that differentiation between good secondary care and CR is artificial. However both of these claims merit further discussion.

The evidence in support of $C R$ remains controversial in the eyes of some. Kotseva et al. refer to compelling scientific support for CR provided by recent large meta-analyses but the more sceptical continue to voice concern about publication bias and the inclusion of many negative and underpowered CR studies. The RAMIT study [West et al. 2011] has to an extent fuelled this controversy by reporting no benefit from CR at 1 and 9 years. This has done little to increase confidence in the value of CRPs for some. A perceived lack of positive data remains a major stumbling block to the engagement of some cardiologists and is perhaps one explanation for a lack of local CR champions. Many cardiologists continue to believe that the observed effects of CR are primarily the consequence of secondary prevention (medication) and that the benefits of short term exercise classes are not maintained. Furthermore it is also often argued that those who are referred to and attend CRPs are the most motivated patients who would 'likely do well anyway'. This view appears to have at least an element of support in the study findings which highlight referral biases which paradoxically appear to lead to the effective exclusion of many potentially suitable participants who it could be argued have most to gain. Thus many cardiologists have been left cold by the results of EUROASPIRE III, reflecting an attitude to CRPs of 'so what it doesn't work anyway' 
Koteseva et al. suggest that the distinction between $\mathrm{CR}$ and secondary prevention is artificial. From a pragmatic perspective we would respectfully disagree. In times of financial austerity coupled with on-going scepticism in some quarters about the efficacy of CR it is crucial that the components that do work are clearly identified and fully evaluated so that potential champions and healthcare funders can make more informed arguments and choices. CR is undoubtedly a complex intervention and research in this area can be challenging but we have a collective duty to determine which components give best value for money and whether this is consistent across patient groups. If it emerges that adherence to pharmacological or non-pharmacological secondary prevention, increased physical activity or psychological support can be delivered using alternative models to traditional CRPs then this requires further evaluation. However given the disappointing results of recent tele-health and self-care initiatives, demonstrating such effects may not be easy [Godlee 2012].

Formal consensus about the constituents of $C R$ is a relatively recent development in the UK [BACPR 2012] and researchers still have much to do in order to evaluate the various components of CR. Standard care has to be defined much more clearly in order to allow meaningful comparison. In an increasingly menu-driven system there also needs to be better understanding and assessment of individual need as is it highly unlikely that one single approach can ever fit all.

Finally, participants often report high levels of satisfaction with CR and views of patients are being given increasing weight by health care commissioners. Understanding the basis for this high level of satisfaction and identification of unmet need in health care systems without CR require further evaluation and may throw useful light on the best way ahead. It may be that alternative solutions can be found in the arena of the internet or social media. Indeed with the recent expansion of health gaming, perhaps we should just consider providing all our patients with an individually tailored Game Boy!

\section{References}

British Association for Cardiovascular Prevention and Rehabilitation (2012) The BACPR Standards and Core Components for Cardiovascular Disease Prevention and Rehabilitation ( $2^{\text {nd }}$ edition) BACPR, UK available from http://www.bacpr.com/resources/15E BACPR Standards FINAL.pdf

Godlee F (2012) Telehealth only part of the solution (Editorial), BMJ 2012:345:e4724

Koteseva K Wood D De Baker G \& De Bacquer D (2012) Use and effects of cardiac rehabilitation in patients with coronary heart disease: results from the EUROASPIRE III Study Group, European Journal of Preventive Cardiology DOI: 10.1177/2047487312449591

West RR Jones DA Henderson AH (2011) Rehabilitation after myocardial infarction Trial (RAMIT): multi-centre randomised controlled trial of comprehensive cardiac rehabilitation in patients following acute myocardial infarction, Heart, 98 (8): 637- 44 